\title{
Bilateral Pyelo-Ureteral Junction Syndrom Complicated with Mute Kidney: About One Case
}

\author{
Kp Avion ${ }^{1}$, N Akassimadou ${ }^{1}$ B Aguia ${ }^{1}$, F Zouan, V Alloka ${ }^{1}$, N Coulibaly ${ }^{2}$, K Dje ${ }^{1}$, A Deby ${ }^{3}$ \\ ${ }^{1}$ Urology Department of the University Hospital Center (CHU) of Bouaké (IvoryCoast) \\ ${ }^{2}$ Urology Department of the CHU University Hospital Center of Bouaké (IvoryCoast) \\ ${ }^{3}$ Public health service of CHU University Hospital of Bouaké (IvoryCoast)
}

*Corresponding Author: Kp Avion, Urology Department of the University Hospital Center (CHU) of Bouaké (IvoryCoast), E-mail: avionkouassi@yahoo.fr

\begin{abstract}
The destruction of the kidney following a pyelo-ureteral junction (JPU) disease is rare. Most often, witness a long evolution of the pathology. The authors report a case of bilateral pyelo-ureteral junction disease complicated by mute left kidney. Treatment consisted of left nephrectomy. The postoperative course was simple. The patient was discharged from hospital on postoperative day 13. The course of the right pyeloureteral junction was performed by the double probe J. The patient was lost of sight after the insertion of this double J probe The diagnostic and therapeutic aspects are discussed by the authors.
\end{abstract}

Keywords: JPU - silent kidney - nephrectomy

\section{INTRODUCTION}

The syndrome of junction pyelo-ureteral (SJPU) is an obstruction of pace often functional, more rarely anatomical of origin generally congenital, it is responsible for a dilation pyélo-caliciel secondary to a permanent or intermittent defect of the evacuation of pyelic urine, sometimes with repercussions on the renal parenchyma [1].

This congenital pathology is the most common cause of renal hydronephrosis, whose diagnosis is often evoked on antenatal ultrasound and confirmed on postnatal examinations in infants [1]. However, delayed diagnosis of pyelo- ureteral junction syndrome can lead to formidable complications such as kidney stones infections and renal failure [2].

We report a rare case of bilateral pyelo-ureteral junction syndrome complicated by mute kidney with intraoperative discovery.

\subsection{Case}

This is a 16-year-old patient with no particular antecedent. He consulted for painful left lumbar mass evolving since 2 months before his admission without notion of hematuria. The clinical examination had found

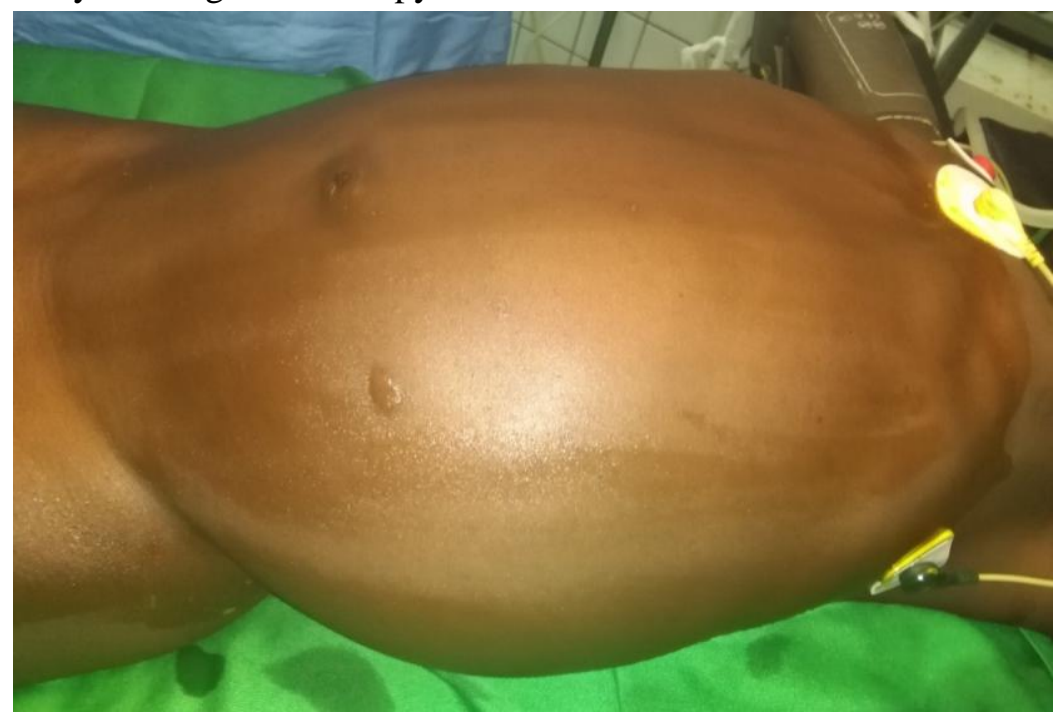

Fig1. Large left lumbar mass deforming the abdomen 
- A lumbar lumbar mass extending from the lumbar region to the side, deforming the abdomen (fig.1).

- Giving lumbar contact.

- There was kidney bouncing

- She measured $18 \mathrm{~cm} / 10 \mathrm{~cm}$

- Smooth, regular surface, mobile in relation to the superficial and deep surface.
- The right lumbar fossa was clinical and normal

- The patient was in good general condition.

We suspected a kidney tumor. The uroscanner revealed a bilateral uretero-hydronephrosis, bulky on the left compressing the abdominal viscera of the neighborhood with an absence of secretion of the kidney. (Fig2 and 3)

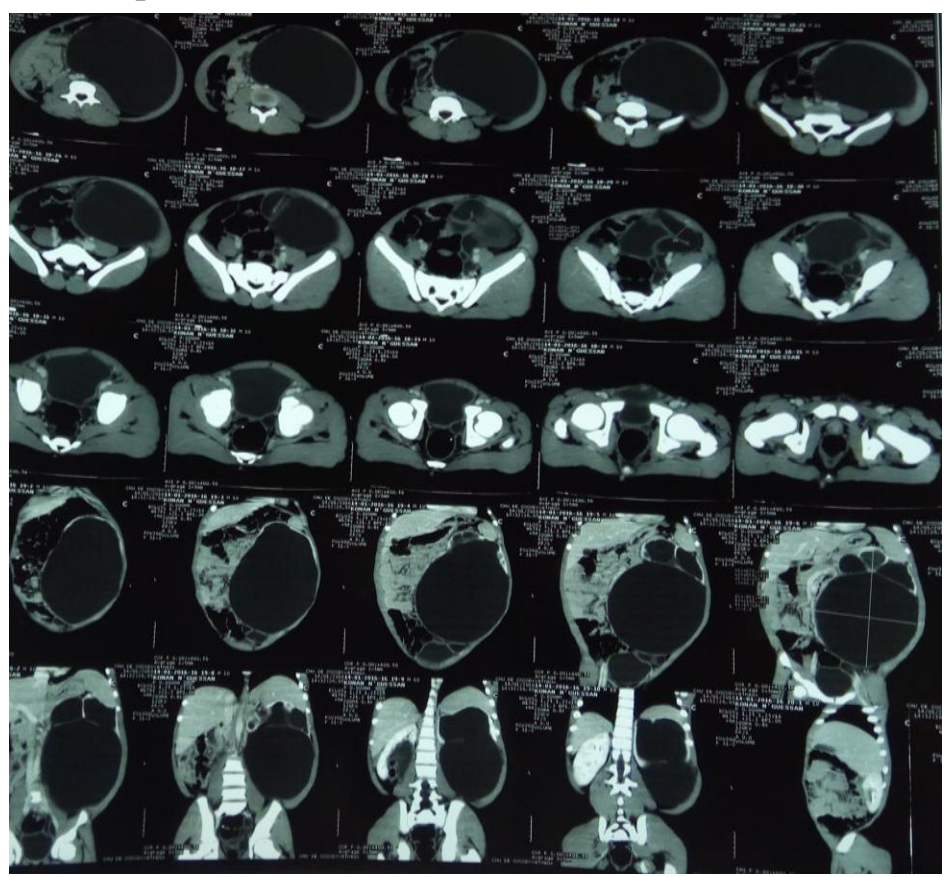

Fig2. Image of left giantureterohydronephrosis on a CT scan

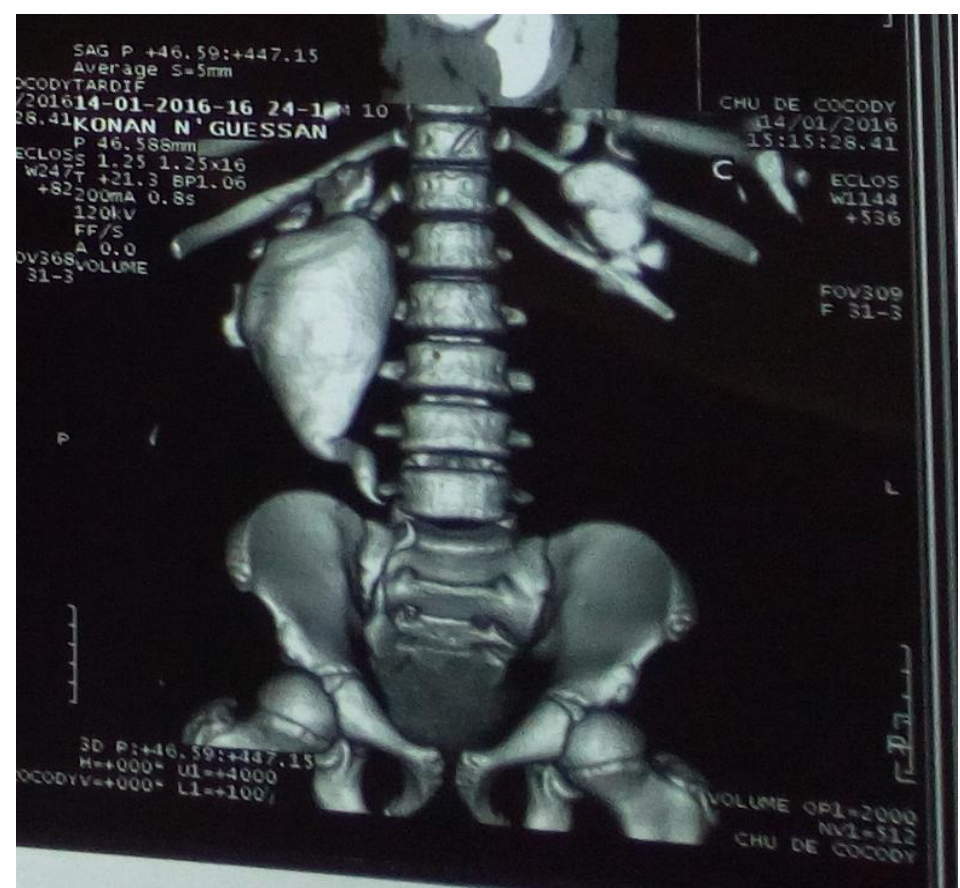

Fig3. Right ureterohydronéphrose plus left dumb kidney (non-secreting) on uroscanner

Before this voluminous uretero-hydro-nephrosis painful with a left dumb kidney, the operative indication had been posed.
Peroperatively, it was a bilateral pyelo-ureteral junction syndrome complicated by a large uretero-hydronephrosis with destruction of the left kidney (Fig.4). The right kidney washealthy. 


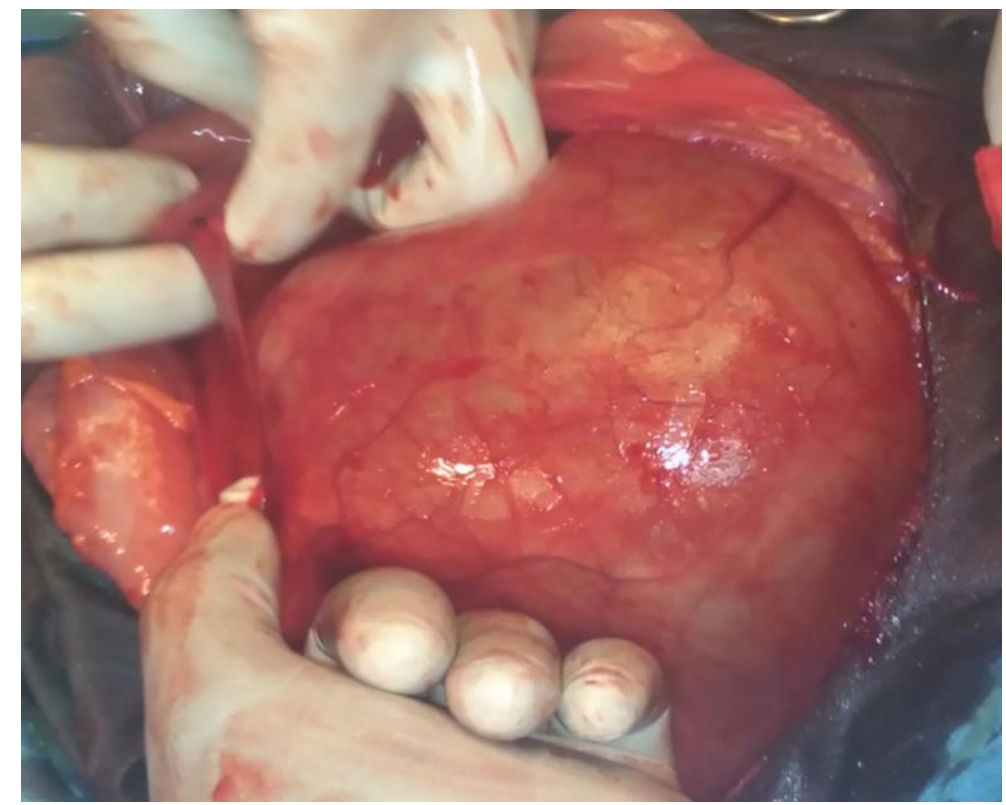

Fig4. Intraoperative view of the pyelo-ureteral junction and hydronephrosis upstream of the junction We performed a left nephro-ureterectomy. (Fig.5)

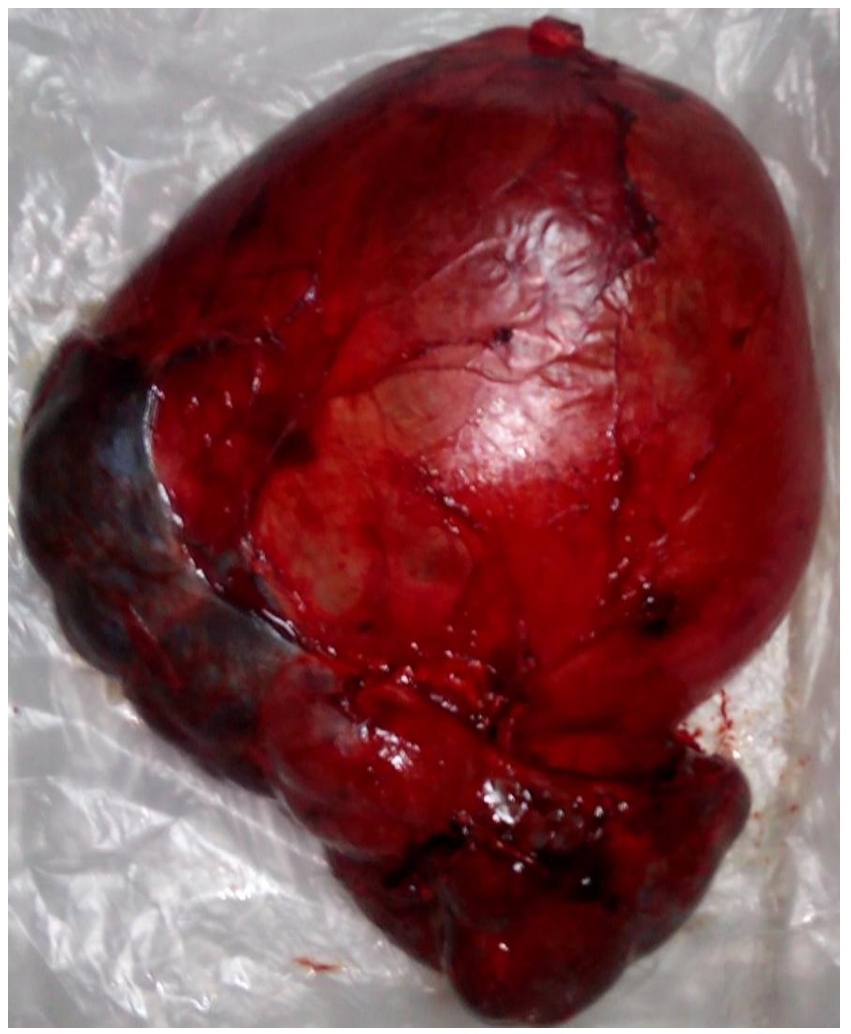

Fig5. Postoperative view of the room after left nephro-ureterectomy

The postoperative course was simple and the patient was discharged on D13 post-operatively. The course of the right pyelo-ureteral junction was performed by the double $\mathrm{J}$ catheter. The patient was lost from view after the placement of this double J probe.

\section{DISCUSSION}

Pyelo-ureteral junction syndrome is a clinical entity that can be congenital or acquired. It causes a distension upstream of the obstacle following a functional or organic obstruction with repercussions on the renal function.

The distention of the upper urinary tract following a sequestration of urine is a source of complication reported by the literature [1]. These are infections, kidney stones, kidney failure. The destruction of the kidney following a pyelo-urethral junction syndrome is rare and our case seems to be the first reported. It could 
be explained by the long evolution of the pathology on the one hand and on the other hand the indolent nature of this affection.

It is a condition that manifests itself at any age from intra-uterine life to the neonatal period to adulthood $[3,4,5]$

In developed countries, accessibility to ultrasound in pregnancy surveillance can detect and monitor antenatal forms. This opportunity offers little in our working conditions given the lack of financial means of patients. This finding reflects the delay in diagnosis especially when the condition is low-noise and asymptomatic as is the case in our patient.

In our case, the patient had bilateral predominant uretero-hydronephrosis on the left. These forms are rare and the literature confirms this rarity. Kiraet al report 2 cases in a series of 35 patients; Diao et al; 5 out of 30 patients.

From a diagnostic point of view, the authors report classic recurrent lumbar pain, exacerbated by heavy drinking, as the most common reason for consultation (91\%) [6].

In our case, the patient consulted for lumbar mass and uroscanner allowed us to highlight this bilateral dilatation of the upper urinary system predominant on the left with mute homolateral kidney. This table is the result of long evolution and delay in consultation. Open surgery was our only recourse for the care of the patient. This is the left nephro-ureterectomy.

Our action was motivated by imaging (uroscanner) and intraoperative findings (nonexistent renal parenchyma). These arguments would have been supported by scintigraphy by giving objective evidence of kidney destruction. However, given the insufficient technical platform coupled with patients' financial difficulties, some advanced diagnostic tests can not be performed.

Nowadays, minimally invasive or laparoscopic endoscopic surgery is increasingly taking precedence over open surgery. However, this technique is not accessible in our practices.

\section{CONCLUSiON}

Pyelo-ureteral junction syndrome is a serious congenital condition due to its insidious course that may be responsible for the destruction of the kidney. The mute kidney must now start from the progressive complications of the pyeloureteral junction syndrome.

\section{REFERENCES}

[1] [1] Descotes JL. Traitement des sténoses de la jonction pyélo-urétérale de l'adulte. Prog urol. 2013; 23: 1172-1176 [Pub Med]

[2] [2] Tembely A, Kassogue A, Berthé H, et al. Aspects cliniques et thérapeutiques des anomalies de la jonction pyélo-urétérale au chu du point G. Pan Afr Med J. 2016 ; 23 : 256. [Pub Med]

[3] [3] Williams B, Tareen B, Resnick MI, etal.pathophysiology and treatment of ureteropelvic junction obstruction. Curr Urol Rep 2007; 8 (2): 111-7. [Pub Med]

[4] [4] DiaoB, Fall B, Kabore FA, et al. AndersonHynes open pyloplasty: which indications in the area of laparoscopic surgery? Prog Urol 2012; 2 (16): 1010-4. [Pub Med]

[5] [5] Tan BJ, Rastinehad AR, Marcovich R. Trends in ureteropelvic junction management among urologists in the United States. Urology 2005; 65 (2): 260-4. [Pub Med]

[6] [6] Kirakoya B, Kabore FA, Zango B, et al. Prise en charge du syndrome de jonction pyélourétérale dans le service d'urologie du centre hospitalier universitaire YalgadoOuedraogo (Burkina Faso) UroAndro 2015 ; 1 : 148-152. [Google scholar]

Citation: Kp Avion1, et.al. Bilateral Pyelo-Ureteral Junction Syndrom Complicated with Mute Kidney: About One Case. ARC Journal of Urology.2019; 4(2):15-18.doi:dx.doi.org/10.20431/2456-060X.0402004.

Copyright: (C) 2019 Authors. This is an open-access article distributed under the terms of the Creative Commons Attribution License, which permits unrestricted use, distribution, and reproduction in any medium, provided the original author and source are credited. 